\begin{tabular}{|c|c|c|c|c|c|c|c|c|}
\hline \multicolumn{2}{|c|}{ Table 7.1} & \multicolumn{7}{|c|}{ Production of compound feeding stuffs } \\
\hline \multicolumn{8}{|c|}{ Monthly averages } & Thousand tonnes \\
\hline & & $\begin{array}{l}\text { Cattle and } \\
\text { calf feed }\end{array}$ & Pig feed & Poultry feed & Sheep feed & Horse feed & Other compounds & Total \\
\hline \multirow{12}{*}{2007} & & BFFB & BFFD & BFFE & JJ8R & JJ8S & BFFF & BFFA \\
\hline & Feb & 333.9 & 120.7 & 206.0 & 110.4 & 17.4 & 21.5 & 810.0 \\
\hline & Mar & 384.4 & 139.3 & 248.2 & 153.6 & 19.9 & 21.5 & 966.9 \\
\hline & Apr & 305.0 & 123.2 & 217.4 & 79.6 & 14.8 & 22.5 & 762.4 \\
\hline & May & 239.2 & 126.8 & 230.7 & 32.1 & 13.4 & 26.5 & 668.7 \\
\hline & Jun & 249.1 & 145.0 & 269.9 & 25.1 & 11.9 & 33.0 & 734.0 \\
\hline & Jul & 268.8 & 133.0 & 259.2 & 21.1 & 12.5 & 34.0 & 728.6 \\
\hline & Aug & 294.2 & 134.5 & 251.8 & 25.1 & 13.0 & 36.2 & 754.7 \\
\hline & Sep & 322.6 & 147.1 & 253.8 & 25.0 & 15.7 & 36.3 & 800.6 \\
\hline & Oct & 332.1 & 137.7 & 237.1 & 24.5 & 15.9 & 30.2 & 777.6 \\
\hline & Nov & 341.3 & 132.2 & 237.4 & 31.8 & 18.5 & 25.9 & 787.2 \\
\hline & Dec & 395.2 & 144.2 & 253.8 & 51.8 & 20.6 & 25.3 & 890.9 \\
\hline \multirow[t]{12}{*}{2008} & Jan & 352.8 & 123.6 & 222.5 & 84.5 & 20.2 & 21.8 & 825.5 \\
\hline & Feb & 353.5 & 116.2 & 215.7 & 109.3 & 18.7 & 22.7 & 836.1 \\
\hline & Mar & 392.1 & 132.5 & 248.2 & 142.3 & 20.3 & 24.4 & 959.8 \\
\hline & Apr & 368.7 & 118.3 & 225.2 & 115.3 & 19.2 & 21.8 & 868.4 \\
\hline & May & 245.1 & 114.9 & 231.9 & 29.9 & 12.8 & 27.4 & 662.0 \\
\hline & Jun & 250.6 & 125.9 & 270.7 & 23.1 & 11.5 & 25.6 & 707.4 \\
\hline & $\mathrm{Jul}^{1}$ & 256.8 & 119.0 & 265.2 & 18.6 & 11.9 & 28.2 & 699.6 \\
\hline & Aug & 262.9 & 115.1 & 240.1 & 17.4 & 11.6 & 31.9 & 679.0 \\
\hline & Sep & 319.9 & 130.7 & 263.8 & 26.0 & 16.9 & 38.3 & 795.5 \\
\hline & Oct & 333.9 & 118.7 & 238.8 & 31.6 & 15.8 & 32.9 & 771.9 \\
\hline & Nov & 321.2 & 112.8 & 227.5 & 43.7 & 16.7 & 27.1 & 749.0 \\
\hline & Dec & 382.9 & 128.9 & 251.4 & 63.6 & 21.6 & 27.5 & 875.9 \\
\hline \multirow[t]{5}{*}{2009} & Jan & 327.3 & 104.7 & 210.8 & 91.3 & 17.8 & 24.9 & 776.8 \\
\hline & Feb & 315.5 & 101.8 & 198.7 & 112.8 & 17.2 & 23.8 & 769.9 \\
\hline & Mar & 369.5 & 116.9 & 242.6 & 142.1 & 19.1 & 18.7 & 908.9 \\
\hline & Apr & 295.4 & 106.4 & 221.2 & 82.6 & 15.7 & 24.5 & 745.8 \\
\hline & May & 249.4 & 105.4 & 229.8 & 36.4 & 11.8 & 27.8 & 660.5 \\
\hline
\end{tabular}

1 Revisions are possible from July 2008

Source: Department for Environment, Food and Rural Affairs: 01904455803

\title{
Table 7.2
}

Animals slaughtered

Monthly averages, totals for four or five week periods and quarters

\begin{tabular}{|c|c|c|c|c|c|c|c|c|}
\hline & \multicolumn{7}{|c|}{ Animals slaughtered (thousands) } \\
\hline & & $\begin{array}{l}\text { Steers, heifers } \\
\text { and young bulls }\end{array}$ & $\begin{array}{l}\text { Cows and } \\
\text { adult bulls }\end{array}$ & Calves & $\begin{array}{c}\text { Ewes and } \\
\text { rams }\end{array}$ & $\begin{array}{l}\text { Other sheep } \\
\text { and lambs }\end{array}$ & $\begin{array}{c}\text { Sows and } \\
\text { adults boars }^{2}\end{array}$ & Clean pigs \\
\hline \multirow{13}{*}{2009} & & BFHA & BFHB & BFHC & BFHD & BFHE & BFHF & BAKP \\
\hline & Jan & 187.1 & 50.0 & 3.4 & 206.8 & $1,259.1$ & 19.0 & 815.8 \\
\hline & Feb & 159.1 & 38.6 & 3.0 & 170.3 & 907.8 & 16.1 & 674.1 \\
\hline & Mar & 155.8 & 35.5 & 3.7 & 172.2 & 890.8 & 15.8 & 651.5 \\
\hline & Apr & 194.8 & 40.1 & 3.2 & 200.7 & $1,057.5$ & 19.2 & 795.9 \\
\hline & May & 155.7 & 33.7 & 2.2 & 149.8 & 762.1 & 14.6 & 624.3 \\
\hline & Jun & 157.4 & 35.4 & 2.6 & 154.7 & 837.5 & 14.3 & 667.3 \\
\hline & Jul & 180.0 & 43.7 & 3.9 & 201.0 & $1,244.8$ & 19.9 & 844.5 \\
\hline & Aug & 137.7 & 34.7 & 3.6 & 182.7 & $1,191.0$ & 16.2 & 688.8 \\
\hline & Sep & 157.2 & 41.1 & 4.3 & 172.0 & $1,202.8$ & 17.0 & 692.5 \\
\hline & Oct & 209.0 & 56.8 & 5.7 & 213.7 & $1,525.4$ & 22.0 & 908.7 \\
\hline & Nov & 172.3 & 49.1 & 3.9 & 191.5 & $1,233.3$ & 17.3 & 739.8 \\
\hline & Dec & 157.8 & 40.9 & 3.3 & 152.6 & $1,101.7$ & 15.2 & 721.0 \\
\hline \multirow[t]{12}{*}{2010} & Jan & 181.6 & 46.2 & 2.9 & 150.0 & 959.0 & 20.1 & 844.3 \\
\hline & Feb & 162.5 & 44.5 & 3.5 & 138.8 & 756.5 & 17.7 & 702.2 \\
\hline & Mar & 164.6 & 39.3 & 4.3 & 132.1 & 818.6 & 18.0 & 707.3 \\
\hline & Apr & 202.2 & 44.3 & 4.2 & 163.4 & 927.9 & 21.1 & 858.3 \\
\hline & May & 162.6 & 37.0 & 2.8 & 132.9 & 700.9 & 16.3 & 694.2 \\
\hline & Jun & 161.5 & 36.4 & 3.0 & 138.2 & 763.8 & 15.2 & 684.9 \\
\hline & Jul & 195.7 & 49.8 & 5.0 & 189.3 & $1,223.0$ & c & 857.0 \\
\hline & Aug & 153.7 & 39.0 & 5.4 & 172.1 & $1,146.3$ & c & 722.2 \\
\hline & Sep & 167.4 & 45.1 & 7.3 & 164.8 & $1,132.2$ & c & 735.3 \\
\hline & Oct & 217.6 & 65.2 & 9.7 & 204.6 & $1,419.1$ & c & 949.5 \\
\hline & Nov & 177.4 & 63.3 & 7.3 & 189.5 & $1,223.2$ & c & 789.9 \\
\hline & Dec & 189.0 & 54.2 & 6.3 & 195.6 & $1,252.8$ & c & 895.6 \\
\hline \multirow[t]{2}{*}{2011} & Jan & 209.8 & 68.0 & 6.7 & 196.3 & $1,023.7$ & c & 905.4 \\
\hline & Feb & 169.9 & 49.2 & 7.5 & 159.1 & 827.3 & c & 766.5 \\
\hline
\end{tabular}

$c$ is confidential data

Source: Department for Environment, Food and Rural Affairs: 01904455803 
Monthly averages, totals for four or five week periods and quarters

\begin{tabular}{|c|c|c|c|c|c|c|}
\hline & \multicolumn{5}{|c|}{ Meat - UK supplies \& Domestic Usage ${ }^{1}$ (thousand tonnes) } \\
\hline & & Beef and veal & Mutton and lamb & Pig meat & Poultry & All Meat \\
\hline & & BFHK & $\mathrm{BFHL}$ & K8D3 & JYXE & KEJ6 \\
\hline 2007 & $\begin{array}{l}\text { Q1 } \\
\text { Q2 } \\
\text { Q3 } \\
\text { Q4 }\end{array}$ & $\begin{array}{l}272.8 \\
274.0 \\
262.0 \\
281.7\end{array}$ & $\begin{array}{r}95.4 \\
104.8 \\
95.5 \\
94.5\end{array}$ & $\begin{array}{l}365.1 \\
363.7 \\
362.8 \\
359.4\end{array}$ & $\begin{array}{l}404.1 \\
400.6 \\
396.3 \\
430.3\end{array}$ & $\begin{array}{l}1,137.4 \\
1,143.0 \\
1,116.5 \\
1,165.9\end{array}$ \\
\hline 2008 & $\begin{array}{l}\text { Q1 } \\
\text { Q2 } \\
\text { Q3 } \\
\text { Q4 }\end{array}$ & $\begin{array}{l}264.4 \\
265.0 \\
263.7 \\
267.6\end{array}$ & $\begin{array}{l}97.7 \\
97.7 \\
89.8 \\
87.2\end{array}$ & $\begin{array}{l}355.6 \\
344.3 \\
333.0 \\
331.3\end{array}$ & $\begin{array}{l}394.1 \\
391.7 \\
392.2 \\
411.9\end{array}$ & $\begin{array}{l}1,111.8 \\
1,098.8 \\
1,078.7 \\
1,098.0\end{array}$ \\
\hline 2009 & $\begin{array}{l}\text { Q1 } \\
\text { Q2 } \\
\text { Q3 } \\
\text { Q4 }\end{array}$ & $\begin{array}{l}246.4 \\
252.2 \\
238.7 \\
270.8\end{array}$ & $\begin{array}{l}92.6 \\
93.2 \\
82.2 \\
82.2\end{array}$ & $\begin{array}{l}332.9 \\
338.4 \\
329.6 \\
361.2\end{array}$ & $\begin{array}{l}392.5 \\
395.7 \\
399.5 \\
436.8\end{array}$ & $\begin{array}{l}1,064.4 \\
1,079.5 \\
1,050.0 \\
1,151.0\end{array}$ \\
\hline 2010 & $\begin{array}{l}\text { Q1 } \\
\text { Q2 } \\
{ }^{1} \\
\text { Q3 } \\
\text { Q4 }\end{array}$ & $\begin{array}{l}255.4 \\
260.4 \\
259.9 \\
275.7\end{array}$ & $\begin{array}{l}80.8 \\
78.5 \\
77.0 \\
76.6\end{array}$ & $\begin{array}{l}340.1 \\
337.0 \\
331.6 \\
344.7\end{array}$ & $\begin{array}{l}423.2 \\
438.5 \\
435.4 \\
482.1\end{array}$ & $\begin{array}{l}1,099.6 \\
1,114.4 \\
1,103.9 \\
1,179.1\end{array}$ \\
\hline
\end{tabular}

1 Data are provisional

\begin{tabular}{lll}
\hline Table 7.4 & Production of pig meat, beef and veal, mutton and lamb and poultrymeat \\
&
\end{tabular}

\begin{tabular}{|c|c|c|c|c|c|c|}
\hline \multicolumn{6}{|c|}{ Meats produced, quarterly averages } & \multirow{2}{*}{$\begin{array}{c}\text { Thousand tonnes } \\
\text { All Meat } \\
\text { Home production }\end{array}$} \\
\hline & & $\begin{array}{c}\text { Pig Meat }{ }^{2} \\
\text { Home production }\end{array}$ & $\begin{array}{c}\text { Beef and veal }{ }^{2} \\
\text { Home production }\end{array}$ & $\begin{array}{l}\text { Mutton and lamb } \\
\text { Home production }\end{array}$ & $\begin{array}{c}\text { Poultry Meat } \\
\text { Home cured production }\end{array}$ & \\
\hline \multirow{2}{*}{\multicolumn{2}{|c|}{2002}} & K8BY & IFY9 & IFZ2 & IFZ3 & JJ8X \\
\hline & & 774 & 694 & 307 & 1,557 & 3,333 \\
\hline \multicolumn{2}{|l|}{2003} & 688 & 703 & 310 & 1,557 & 3,258 \\
\hline \multicolumn{2}{|l|}{$2004^{4}$} & 679 & 724 & 319 & 1,543 & 3,265 \\
\hline \multicolumn{2}{|l|}{2005} & 669 & 765 & 337 & 1,570 & 3,341 \\
\hline \multicolumn{2}{|l|}{2006} & 668 & 852 & 334 & 1,512 & 3,365 \\
\hline \multicolumn{2}{|l|}{$2007^{3}$} & 707 & 889 & 330 & 1,466 & 3,391 \\
\hline \multicolumn{2}{|l|}{2008} & 706 & 866 & 332 & 1,464 & 3,368 \\
\hline \multicolumn{2}{|l|}{$2009^{3}$} & 681 & 841 & 309 & 1,459 & 3,290 \\
\hline \multirow[t]{3}{*}{2005} & Q2 & 163 & 191 & 71 & 381 & 806 \\
\hline & Q3 & 165 & 191 & 89 & 390 & 835 \\
\hline & Q4 & 174 & 204 & 97 & 397 & 872 \\
\hline \multirow[t]{4}{*}{2006} & Q1 & 165 & 207 & 84 & 373 & 828 \\
\hline & Q2 & 158 & 207 & 70 & 382 & 817 \\
\hline & Q3 & 168 & 209 & 88 & 370 & 835 \\
\hline & Q4 & 177 & 229 & 93 & 387 & 886 \\
\hline \multirow[t]{4}{*}{2007} & Q1 & 174 & 228 & 76 & 363 & 841 \\
\hline & Q2 & 171 & 220 & 76 & 364 & 831 \\
\hline & Q3 & 170 & 210 & 82 & 361 & 822 \\
\hline & Q4 & 192 & 231 & 95 & 378 & 897 \\
\hline \multirow[t]{4}{*}{2008} & Q1 & 181 & 222 & 84 & 359 & 846 \\
\hline & Q2 & 171 & 214 & 68 & 359 & 813 \\
\hline & Q3 & 175 & 208 & 87 & 368 & 837 \\
\hline & Q4 & 179 & 223 & 93 & 378 & 872 \\
\hline \multirow[t]{4}{*}{2009} & $\mathrm{Q1}^{3}$ & 165 & 208 & 72 & 354 & 799 \\
\hline & $\mathrm{Q}^{3}$ & 160 & 204 & 66 & 357 & 786 \\
\hline & $\mathrm{Q}^{3}$ & 171 & 190 & 86 & 360 & 807 \\
\hline & $\mathrm{Q} 4^{3}$ & 185 & 222 & 89 & 387 & 883 \\
\hline \multirow[t]{4}{*}{2010} & $\mathrm{Q} 1^{3}$ & 176 & 217 & 61 & 375 & 828 \\
\hline & $\mathrm{Q}^{3}$ & 171 & 220 & 58 & 378 & 827 \\
\hline & $\mathrm{Q}^{3}$ & 177 & 221 & 82 & 384 & 864 \\
\hline & $\mathrm{Q} 4^{3}$ & 189 & 241 & 87 & 435 & 950 \\
\hline
\end{tabular}

1 Does not include meat offals or trade in preserved or manufactured meat products (eg: canned meat).

2 Includes an estimate of the dressed carcase weight of live exports other than pure bred.

3 Data are provisional.

42004 home-fed production figures were collected on a 53 week (14 week 4th Quarter) basis but have been adjusted to a 52 week year (13 week 4th quarter) for comparability.

Source: Department for Environment, Food and Rural Affairs: 01904455803 


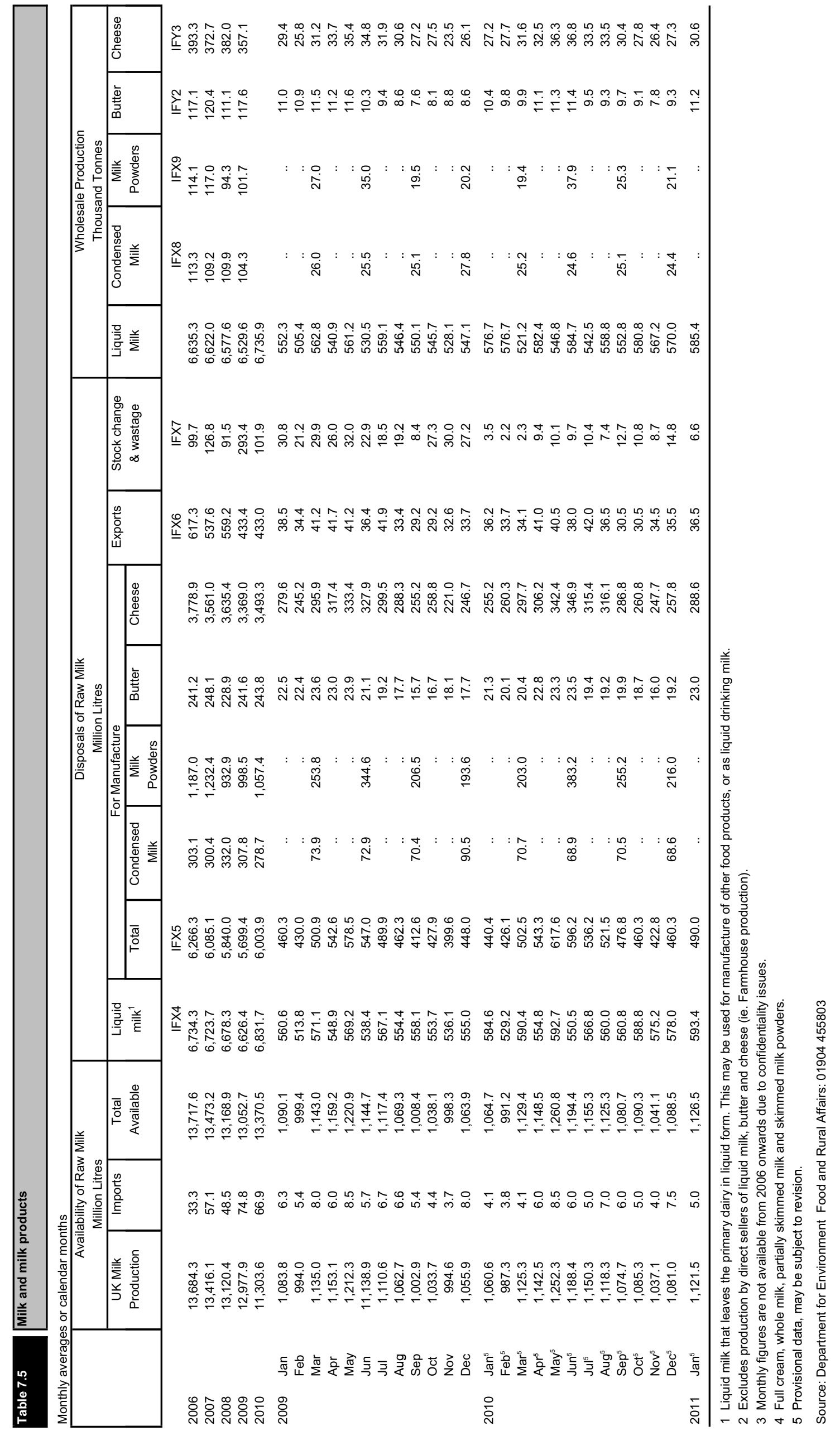


Monthly averages or totals for four or five week periods. Stocks refer to the end of the period

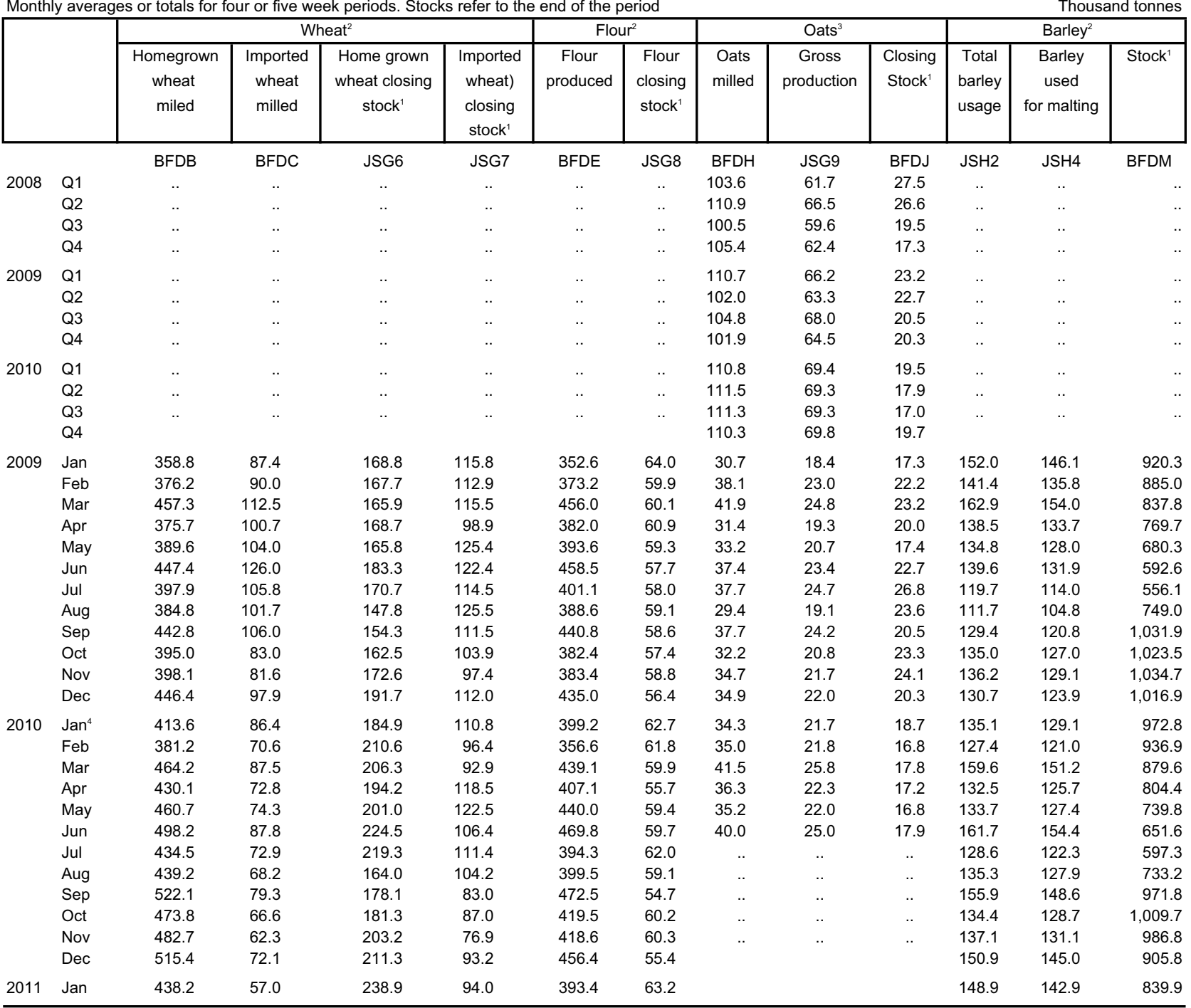

Note - Due to rounding totals may not agree with the sum of the individual items.

1 Stocks held by wheat millers, feed compounders, cereal breakfast food manufacturers, brewers, maltsters and distillers, merchants and dealers.

2 Figures for Wheat, Flour and Barley are only available monthly.

3 Monthly figures for Oats are not available after June 2010.

4 There were 53 weeks in the Statistical year 2010. In order to incorporate the change January 2010 was increased to

a 5 week period compared to 4 weeks in 2009.

Source: Department for Environment, Food and Rural Affairs: 01904455803 
Tobacco products released for home consumption

Monthly averages or calendar months

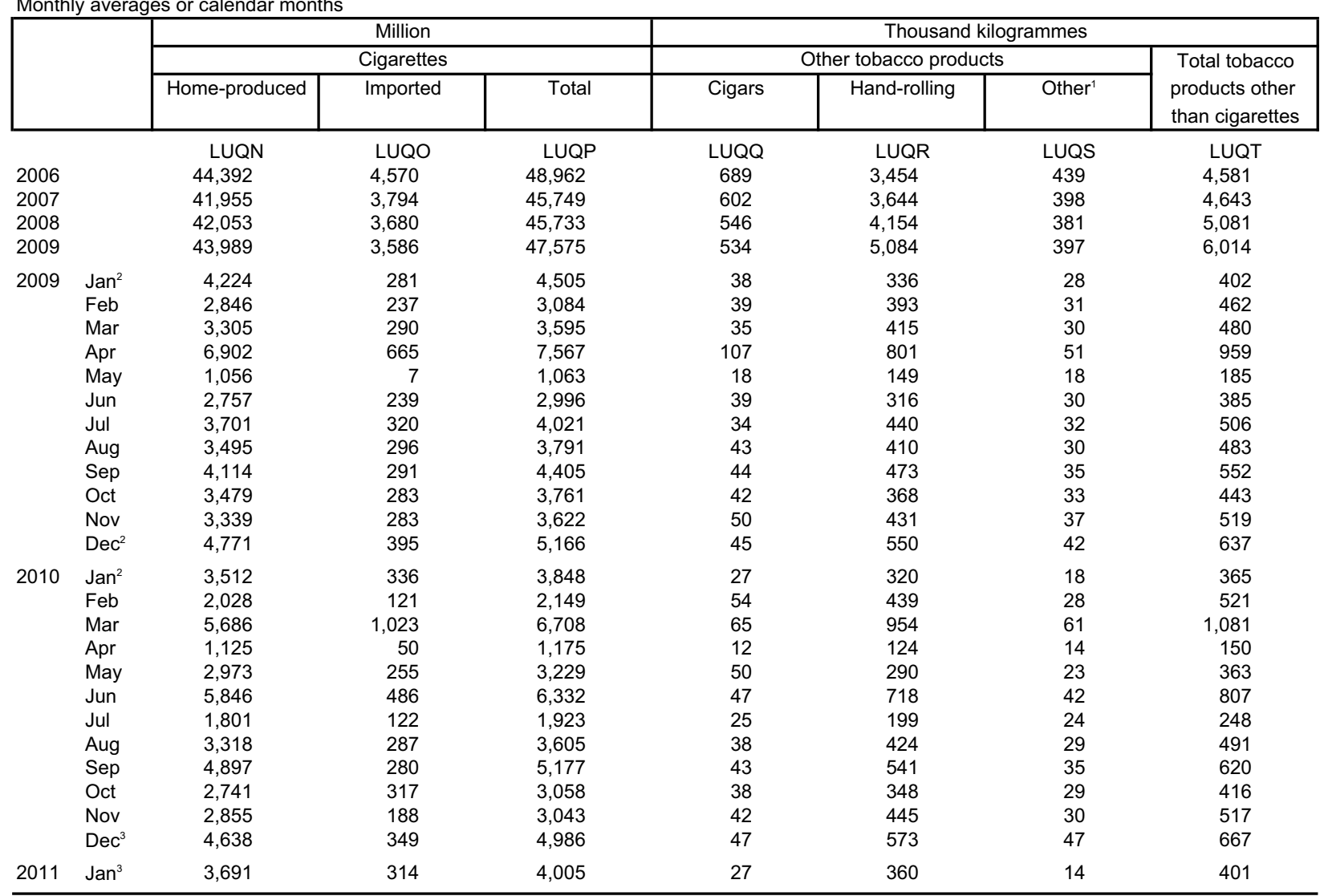

Note: Releases of cigarettes and other tobacco products tend to be higher in the period before a Budget.

Products may then be stocks, duty paid, before being sold.

1 Excluding snuff.

2 Due to the effect of the holiday period, these figures are subject to greater uncertainty than usual.

3 Provisional

Sources: HM Revenueand Customs Statistical Bulletins at; http://www.uktradeinfo.com/index.cfm?task=bulletins 


\begin{tabular}{|c|c|c|c|c|c|c|c|c|c|c|c|c|c|c|c|}
\hline \multicolumn{2}{|c|}{ Table 7.8} & \multicolumn{14}{|c|}{ Alcoholic drink } \\
\hline & & \multicolumn{9}{|c|}{ Thousand hectolitres } & \multicolumn{5}{|c|}{ Thousand hectolitres of alcohol } \\
\hline & & \multirow{4}{*}{$\begin{array}{l}\text { Beer } \\
\text { produc- } \\
\text { tion }\end{array}$} & & & Relea & ased for hor & ne consum & ption & & & Released $\mathrm{f}$ & r home col & iumption & Production 0 & ble spirits ${ }^{1}$ \\
\hline & & & Beer & & Wine of fre & esh grapes & & Made & vine & Cider & & Spirits & & Home- & Other \\
\hline & & & & Not exceec & ding $15 \%$ & Over $15 \%$ & Total & Coolers $^{3}$ & Other & and & Home- & Spirit- & Other $^{5}$ & produced & \\
\hline & & & & Still & Sparkling & ABV & & & & perry & $\begin{array}{c}\text { produced } \\
\text { whisky }\end{array}$ & $\begin{array}{c}\text { based } \\
\text { coolers }^{4} \\
\end{array}$ & & whisky & \\
\hline & & BFNK & BAYL & BFNO & BFNS & BFNP & BFNT & BAYM & BAYN & BFNW & BFNX & YZUJ & BFNY & BAYO & BAYP \\
\hline 2006 & & 53,768 & 55,751 & $11,655.4$ & 715.0 & 301.5 & $12,671.9$ & 527.6 & 316.7 & $7,522.6$ & 282.6 & 64.9 & 766.9 & $3,985.7$ & 499.5 \\
\hline 2007 & & 51,341 & 53,465 & $12,559.1$ & 838.1 & 305.0 & $13,702.2$ & 720.1 & 348.0 & $8,046.0$ & 285.9 & 51.8 & 832.2 & $5,059.5$ & 439.0 \\
\hline 2008 & & 49,611 & 51,498 & $12,402.2$ & 757.4 & 323.9 & $13,483.5$ & 611.5 & 381.5 & $8,412.3$ & 289.4 & 41.7 & 816.6 & $5,516.1$ & 556.1 \\
\hline 2009 & & 45,141 & 46,817 & $11,729.3$ & 731.3 & 219.1 & $12,679.8$ & 596.8 & 392.5 & $9,403.8$ & 258.1 & 31.8 & 801.6 & $4,855.0$ & 901.8 \\
\hline & & BFNK & BAYL & BFNO & BFNS & BFNP & BFNT & BAYM & BAYN & BFNW & BFNX & YZUJ & BFNY & BAYO & BAYP \\
\hline 2006 & Jan & 3,564 & 3,157 & 693.6 & 46.9 & 11.5 & 751.9 & 24.8 & 19.7 & 359.4 & 13.0 & 3.3 & 32.2 & & \\
\hline & Feb & 3,105 & 3,748 & 843.4 & 41.2 & 14.0 & 898.6 & 31.6 & 16.6 & 437.8 & 16.0 & 3.7 & 42.9 & & \\
\hline & Mar & 5,218 & 5,492 & $1,095.5$ & 41.4 & 18.9 & $1,155.8$ & 38.1 & 31.9 & 527.8 & 21.6 & 5.0 & 59.4 & $1,019.7$ & 140.3 \\
\hline & Apr & 4,029 & 4,005 & 955.0 & 40.5 & 16.9 & $1,012.5$ & 42.8 & 19.2 & 560.1 & 21.8 & 5.6 & 59.8 & & \\
\hline & May & 4,784 & 5,395 & 891.6 & 46.5 & 15.9 & 954.0 & 35.2 & 22.2 & 647.9 & 20.1 & 5.1 & 55.0 & & \\
\hline & Jun & 5,295 & 5,362 & 961.7 & 54.9 & 17.8 & $1,034.4$ & 49.8 & 25.6 & 695.7 & 24.2 & 8.2 & 75.0 & $1,222.8$ & 118.1 \\
\hline & Jul & 4,852 & 4,558 & 980.1 & 57.4 & 13.8 & $1,051.4$ & 50.1 & 24.2 & 753.5 & 16.8 & 4.5 & 50.5 & & \\
\hline & Aug & 4,573 & 4,900 & 999.3 & 49.7 & 17.0 & $1,066.0$ & 49.6 & 25.2 & 849.8 & 21.0 & 7.0 & 64.4 & & \\
\hline & Sep & 4,205 & 4,332 & 969.1 & 53.8 & 21.6 & $1,044.5$ & 39.8 & 27.7 & 690.1 & 20.9 & 4.5 & 61.9 & 909.4 & 110.4 \\
\hline & Oct & 4,667 & 4,740 & 974.9 & 65.7 & 34.8 & $1,075.5$ & 47.6 & 38.1 & 682.5 & 31.6 & 5.7 & 72.8 & & \\
\hline & Nov & 4,053 & 4,436 & $1,242.6$ & 106.9 & 67.5 & $1,417.0$ & 53.7 & 42.9 & 695.3 & 38.2 & 4.9 & 100.5 & & \\
\hline & $\operatorname{Dec}^{6}$ & 5,423 & 5,626 & $1,048.5$ & 110.1 & 51.8 & $1,210.4$ & 64.6 & 23.5 & 622.8 & 37.4 & 7.3 & 92.5 & 833.8 & 130.7 \\
\hline 2007 & $\operatorname{Jan}^{6}$ & 3,241 & 3,221 & 936.1 & 65.0 & 20.1 & $1,021.3$ & 41.7 & 22.5 & 520.1 & 19.1 & 4.5 & 61.5 & & \\
\hline & Feb & 3,303 & 3,530 & 821.5 & 40.3 & 11.8 & 873.7 & 36.8 & 18.2 & 495.3 & 15.7 & 3.1 & 48.5 & & \\
\hline & Mar & 4,926 & 5,342 & $1,059.6$ & 56.1 & 16.7 & $1,132.4$ & 54.4 & 35.7 & 650.1 & 21.6 & 3.9 & 59.6 & $1,265.5$ & 75.2 \\
\hline & Apr & 4,135 & 4,092 & 979.5 & 51.0 & 18.9 & $1,049.4$ & 57.1 & 19.8 & 615.6 & 18.9 & 4.6 & 56.6 & & \\
\hline & May & 4,494 & 4,903 & $1,014.9$ & 48.9 & 15.2 & $1,079.1$ & 56.2 & 26.6 & 726.1 & 21.4 & 4.1 & 63.6 & & \\
\hline & Jun & 4,278 & 4,387 & $1,110.9$ & 64.1 & 18.3 & $1,193.4$ & 71.1 & 23.4 & 797.0 & 22.9 & 4.5 & 65.3 & $1,359.8$ & 91.8 \\
\hline & Jul & 4,388 & 4,180 & 987.7 & 60.0 & 17.1 & $1,064.8$ & 53.9 & 30.3 & 608.0 & 18.5 & 2.9 & 54.8 & & \\
\hline & Aug & 4,643 & 5,307 & $1,263.9$ & 66.9 & 20.8 & $1,351.6$ & 79.9 & 32.0 & 760.5 & 27.2 & 6.3 & 78.0 & & \\
\hline & Sep & 4,056 & 4,057 & 991.0 & 68.5 & 25.3 & $1,084.9$ & 55.6 & 29.0 & 852.2 & 20.3 & 4.1 & 60.1 & $1,181.0$ & 94.1 \\
\hline & Oct & 4,727 & 4,892 & $1,061.0$ & 74.4 & 34.0 & $1,169.5$ & 60.3 & 35.1 & 614.2 & 26.3 & 2.6 & 66.6 & & \\
\hline & Nov & 4,635 & 4,865 & $1,325.3$ & 128.2 & 55.9 & $1,509.4$ & 91.7 & 42.6 & 740.2 & 40.9 & 6.4 & 112.6 & & \\
\hline & $\operatorname{Dec}^{6}$ & 4,515 & 4,689 & $1,007.7$ & 114.5 & 50.6 & $1,172.9$ & 61.4 & 32.7 & 666.7 & 33.0 & 4.8 & 105.1 & $1,253.2$ & 177.8 \\
\hline 2008 & $\operatorname{Jan}^{6}$ & 2,998 & 3,208 & 772.5 & 51.5 & 20.1 & 844.1 & 26.9 & 22.9 & 547.6 & 15.3 & 2.0 & 41.8 & & \\
\hline & Feb & 3,589 & 3,878 & 927.4 & 58.5 & 20.5 & $1,006.3$ & 37.6 & 23.7 & 513.3 & 17.3 & 2.9 & 46.8 & & \\
\hline & Mar & 4,214 & 4,462 & $1,301.1$ & 59.4 & 27.2 & $1,387.7$ & 49.9 & 48.1 & 780.6 & 34.1 & 4.0 & 90.7 & $1,344.6$ & 71.3 \\
\hline & Apr & 4,884 & 4,066 & 800.6 & 39.0 & 14.8 & 854.5 & 41.8 & 18.9 & 542.9 & 10.5 & 1.6 & 43.3 & & \\
\hline & May & 4,486 & 4,770 & 1,103.6 & 57.8 & 37.1 & $1,198.6$ & 59.1 & 29.6 & 995.4 & 24.4 & 5.5 & 72.6 & & \\
\hline & Jun & 4,290 & 4,355 & 967.2 & 58.8 & 7.3 & $1,033.3$ & 42.5 & 30.8 & 619.2 & 19.2 & 3.5 & 60.3 & $1,500.4$ & 175.0 \\
\hline & Jul & 4,082 & 4,264 & $1,032.5$ & 64.9 & 19.2 & $1,116.6$ & 50.8 & 27.3 & 798.1 & 17.0 & 3.3 & 53.8 & & \\
\hline & Aug & 4,233 & 4,241 & $1,073.7$ & 46.3 & 20.6 & $1,140.6$ & 56.5 & 29.2 & 947.4 & 23.0 & 3.5 & 66.2 & & \\
\hline & Sep & 3,909 & 4,353 & 834.6 & 43.5 & 15.2 & 893.3 & 42.6 & 27.7 & 480.5 & 21.2 & 2.9 & 58.8 & $1,250.4$ & 151.2 \\
\hline & Oct & 4,379 & 4,504 & $1,130.2$ & 66.3 & 38.1 & $1,234.6$ & 49.2 & 41.6 & 784.0 & 35.1 & 3.8 & 89.8 & & \\
\hline & Nov & 4,670 & 5,602 & $1,439.2$ & 110.8 & 59.6 & $1,609.6$ & 99.7 & 56.3 & 892.0 & 55.8 & 7.5 & 136.5 & & \\
\hline & $\operatorname{Dec}^{6}$ & 3,877 & 3,795 & $1,019.7$ & 100.5 & 44.2 & $1,164.4$ & 54.7 & 25.3 & 511.5 & 16.3 & 1.3 & 55.9 & $1,420.7$ & 158.7 \\
\hline 2009 & $\operatorname{Jan}^{6}$ & 2,711 & 2,651 & 734.3 & 54.3 & 12.1 & 800.8 & 39.1 & 30.9 & 586.9 & 12.9 & 1.4 & 51.5 & & \\
\hline & Feb & 2,986 & 3,115 & 725.6 & 37.0 & 12.8 & 775.3 & 25.3 & 30.3 & 510.0 & 13.1 & 1.4 & 44.6 & & \\
\hline & Mar & 3,600 & 4,007 & 809.5 & 35.9 & 11.0 & 856.4 & 31.8 & 27.5 & 614.2 & 19.0 & 2.3 & 58.2 & $1,293.3$ & 166.9 \\
\hline & Apr & 4,433 & 4,727 & $1,172.8$ & 49.9 & 13.1 & $1,235.8$ & 51.3 & 41.4 & 812.4 & 28.1 & 4.3 & 87.6 & & \\
\hline & May & 3,651 & 3,801 & 901.6 & 48.9 & 12.7 & 963.2 & 48.3 & 18.5 & 743.1 & 15.1 & 2.0 & 45.9 & & \\
\hline & Jun & 3,744 & 3,968 & 995.3 & 57.3 & 11.8 & $1,064.3$ & 52.3 & 22.4 & 910.1 & 19.1 & 3.3 & 61.0 & $1,284.6$ & 165.6 \\
\hline & Jul & 4,301 & 4,316 & $1,004.2$ & 55.7 & 10.4 & $1,070.3$ & 72.2 & 27.6 & $1,049.0$ & 16.8 & 2.7 & 57.4 & & \\
\hline & Aug & 4,203 & 4,084 & $1,013.7$ & 52.8 & 12.9 & $1,079.4$ & 60.0 & 32.0 & 1,003.9 & 21.0 & 3.6 & 64.9 & & \\
\hline & Sep & 3,811 & 3,771 & 918.3 & 53.3 & 13.5 & 985.1 & 32.3 & 35.4 & 677.2 & 17.8 & 2.3 & 59.9 & $1,066.3$ & 284.3 \\
\hline & Oct & 3,875 & 3,853 & $1,135.5$ & 59.7 & 31.5 & $1,226.7$ & 49.7 & 46.1 & 880.0 & 29.5 & 2.5 & 85.6 & & \\
\hline & Nov & 3,606 & 4,157 & $1,274.9$ & 127.0 & 44.2 & $1,446.1$ & 62.0 & 46.9 & 736.9 & 37.3 & 3.5 & 97.4 & & \\
\hline & $\operatorname{Dec}^{6}$ & 4,220 & 4,367 & $1,043.6$ & 99.5 & 33.2 & $1,176.2$ & 72.5 & 33.6 & 880.1 & 28.3 & 2.6 & 87.6 & $1,210.8$ & 285.0 \\
\hline 2010 & $\operatorname{Jan}^{6}$ & 2,390 & 2,590 & 895.1 & 69.3 & 16.7 & 981.1 & 41.3 & 21.3 & 630.0 & 17.9 & 2.4 & 57.8 & & \\
\hline & Feb & 2,896 & 3,014 & 747.4 & 56.6 & 10.6 & 814.6 & 40.5 & 30.3 & 546.7 & 16.0 & 1.3 & 51.2 & & \\
\hline & Mar & 3,823 & 4,246 & $1,030.1$ & 58.4 & 17.7 & $1,106.2$ & 74.7 & 39.7 & 862.1 & 37.2 & 2.6 & 94.3 & $1,180.9$ & 269.3 \\
\hline & Apr & 4,550 & 3,953 & 977.2 & 47.3 & 11.7 & $1,036.2$ & 49.4 & 25.6 & 803.9 & 14.6 & 5.4 & 57.0 & & \\
\hline & May & 5,275 & 5,306 & 942.4 & 45.5 & 12.6 & $1,000.5$ & 56.7 & 28.4 & 844.0 & 14.3 & 2.6 & 48.3 & & \\
\hline & Jun & 3,509 & 3,775 & $1,059.6$ & 62.7 & 11.3 & $1,133.6$ & 81.7 & 29.4 & $1,342.2$ & 19.7 & 2.8 & 72.7 & $1,230.2$ & 589.6 \\
\hline & Jul & 3,613 & 3,481 & $1,095.5$ & 64.1 & 12.1 & $1,171.6$ & 94.8 & 32.7 & 759.3 & 14.9 & 3.6 & 52.8 & & \\
\hline & Aug & 3,889 & 3,917 & 908.1 & 49.1 & 10.6 & 967.8 & 82.9 & 34.7 & 914.4 & 16.0 & 2.4 & 58.1 & & \\
\hline & $\operatorname{Sep}^{7}$ & 3,690 & 3,535 & 960.8 & 61.2 & 14.2 & $1,036.2$ & 48.7 & 35.3 & 585.1 & 15.7 & 1.7 & 59.8 & $1,006.7$ & 773.0 \\
\hline & Oct & 3,561 & 3,712 & $1,004.1$ & 78.6 & 27.6 & $1,150.3$ & 57.6 & 46.0 & 800.7 & 32.5 & 2.8 & 88.9 & & \\
\hline & Nov & 4,135 & 4,093 & $1,203.3$ & 112.9 & 39.3 & $1,355.5$ & 67.2 & 47.2 & 595.0 & 38.1 & 3.4 & 99.9 & & \\
\hline & $\operatorname{Dec}^{8}$ & 3,666 & 4,251 & $1,006.7$ & 104.1 & 36.0 & $1,146.8$ & 62.0 & 32.1 & 711.8 & 30.0 & 4.6 & 101.0 & $1,237.6$ & 891.8 \\
\hline 2011 & $\mathrm{Jan}^{8}$ & 2,444 & 2,544 & 819.6 & 61.7 & 14.5 & 895.8 & 43.1 & 27.0 & 690.9 & 14.5 & 1.0 & 43.5 & & \\
\hline
\end{tabular}

Note: some figures have seen small revisions since they were last published.

1 Data are available only quarterly and in the months following the quarter.

2 Percentage alcohol by volume.

3 Made wine with alcoholic strength $1.2 \%$ to $5.5 \%$, includes alcoholic lemonade of appropriate strength

4 From 28 April 2002 duty on spirit-based ready-to-drink (RTDs) products is charged at the same rate as spirits per litre of alcohol.

Spirit-based RTDs were previously dutied at the made wine rate and details on quantities can be found in made wine coolers.

5 Includes imported spirits.

6 Due to the effect of the holiday period, these figures are subject to greater uncertainty than usual. Also, unusually high or low figures may be changed on receipt of

amendments to returns data.

7 Revisions are possible from July 2010

8 Provisional

Source: HM Revenue and Customs Alcohols Bulletins at: https://www.uktradeinfo.com/index.cfm?task=bullAlcohol 
Standard Industrial Classification 2003

$2005=100$, seasonally adjusted

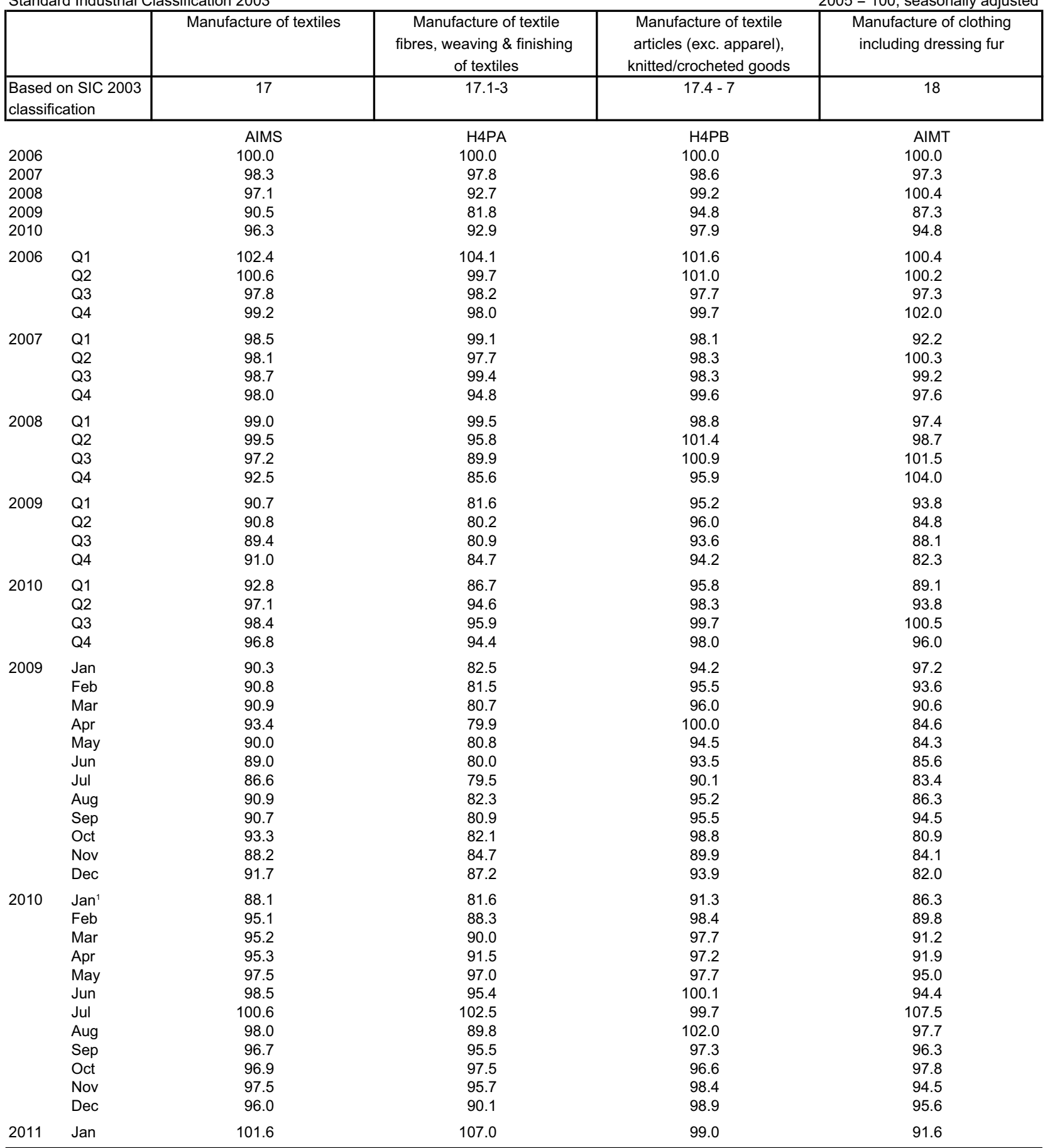

Note: ONS has introduced metholological changes to the Index of Production from March 2008 (January 2008 data). Also, a new industry

structure means that the level of detail published is reduced. For the textile and clothing sector, industries are no longer available at the full level of disaggregation. Further information can be found on the NS website at - http://www.statistics.gov.uk/cci/article.asp?id=1908.

1 Please note that Periods open to revision are from January 2010 this round.

Source: Office for National Statistics: 01633455803 